\title{
Wool Functionalization by Using Green Synthesized Silver Nanoparticles
}

\author{
SAKIL MAHMUD ${ }^{1,2 *}$, MD. NAHID PERVEZ ${ }^{1,3}$, MST. ZAKIA SULTANA $^{1,4}$, \\ MD. AHSAN HABIB ${ }^{1,5}$ and HUI-HONG LIU ${ }^{1 *}$ \\ ${ }^{1}$ School of Chemistry and Chemical Engineering, Wuhan Textile University, \\ Wuhan, P. R. China- 430200. \\ ${ }^{2}$ Ningbo Institute of Material Technology and Engineering, Chinese Academy \\ of Sciences, Ningbo, P.R. China-315201. \\ ${ }^{3}$ Research Institute of Flexible Materials, School of Textiles \& Design, Heriot-Watt \\ University, TD1 3HF, United Kingdom. \\ ${ }^{4}$ NUS Graduate School for Integrative Science \& Engineering, National University of \\ Singapore, 28 Medical Drive, Singapore 117456 , Singapore. \\ ${ }^{5}$ Institutes of Chemistry, Chinese Academy of Sciences, Beijing P. R. China- 100190. \\ ${ }^{*}$ Corresponding author E-mail: sakilhabib@qq.com, huihongliu@126.com,
}

http://dx.doi.org/10.13005/ojc/330507

(Received: July 18, 2017; Accepted: August 25, 2017)

\begin{abstract}
In this study, for the first time wool fabric was functionalized through green based synthesized silver nanoparticles (AgNPs) employing sodium alginate as reducing agent. The diffusion of AgNPs into wool surface as well as wool polymer system seems to be dependent on both physical and chemical interactions. The resulting products were characterized with scanning electron microscopy (SEM), Fourier transform infrared spectroscopy (FT-IR) spectra, X-ray diffraction (XRD), Thermogravimetric analysis (TGA), and UV-vis absorbance. The results specified that AgNPs were successfully assembled on the wool surface when the liquor $\mathrm{pH}$ and temperature of the application medium was adjusted to 4 and $45^{\circ} \mathrm{C}$, respectively for 2 hours. This experiment also indicates that the wool fabric with Ag-NPs shows obvious multifunctional action because of the presence of the Ag-NPs. The treated wool fabrics exhibit optimistic colors due to the localized surface plasmon resonance (SPR) of Ag-NPs. The use of this technique to treat wool fabric may lead to new coloration technique and other functional improvement.
\end{abstract}

Keywords: Green synthesis, AgNPs, Sodium alginate, Wool, Multifunctional

\section{INTRODUCTION}

With the advent of science and technology, a new era has emerged in the realm of textile processing by negotiating of metal nanoparticles due to nanoscale materials and structures, typically ranging from 1 to 100 nanometres $(\mathrm{nm})^{1}$. A significant challenge in the textile industry is that conventional approaches to functionalize fabrics don't prompt perpetual impacts². For example, 
laundering decreases imparted functional effects. Hence, nanoparticles due to their diverse functions have been applied to impart flame retardant, UVblocking, water repellent, self-cleanining, and antimicrobial properties to the textile fibres ${ }^{3,4}$. The advantage of nanomaterials concerns developing function without altering the solace properties of the substrate ${ }^{5}$. Among several inorganic nanomaterials, silver is one of the most important and most studied due to their exceptional antibacterial, antifungal activity, less toxicity and low cost approach ${ }^{6}$. Researchers have devoted unique interest to diverse guidance techniques consisting of gamma irradiation, photo-catalytic reduction, chemical reduction, microwave processing, photo-chemical method, metallic wire explosion, sonochemical, polyols, electron irradiation, and biological methods for the synthesis of silver nanoparticles ${ }^{7}$. As of late expanding request must be joined by "green" synthesis methods ${ }^{8,9}$. In the global efforts to reduce generated hazardous waste, "green" chemistry and chemical processes are steadily integrating with current developments in science and industry. Implementation of these sustainable processes should adopt the fundamental principles of green chemistry. These standards are outfitted to direct in limiting the utilization of unsafe products and maximizing the efficiency of chemical processes. Henceforth, any engineered course or substance process should address these standards by utilizing ecologically favourable solvents and nontoxic chemicals ${ }^{10}$. The green synthesis of AgNPs involves three main steps, which must be assessed in view of green chemistry perspectives, including (1) selection of solvent medium, (2) selection of environmentally benign reducing agent, and (3) selection of nontoxic substances for the Ag NPs stability ${ }^{11}$. Based on this approach, we have produced the green-chemistry type Ag NP synthesis processes in this research. Natural fibers are a decent media for developing diverse microorganism due to giving a good energy source of nutrients. The natural protein fiber wool is a superior natural textile material due to its resilience and comfort attributes. It is additionally an accommodating host for the era and engendering of microorganisms, bringing about fiber harms and even skin disturbances, especially for products such as garments and carpets. Because wool is mainly composed of keratin, the outermost part of the fiber is the cuticle cell, of which the surface is a fatty layer of 18-methyl Eicosanoic acid covalently bound to the protein layer of the wool cuticle via a thioester linkage. Due to the presence of this fatty layer, the surface of wool is hydrophobic ${ }^{12}$. The presence of this unique characteristic makes them ideal for the selective binding of metal ions. Therefore, the wool fiber with polyfunctional ligands can be a suitable template to grow metal nanoparticles. There are several reports in which the wool fabrics have been loaded with metal nanoparticles to impart antibacterial properties ${ }^{13-16}$.

In the present research, we report for the first time that green based AgNPs have been used to functionalize wool fibers employing sodium alginate (NaAlg) as a reducing agent. Synthesized AgNPs were applied to wool fabric by exhaustion which involves in three different steps i.e., initially wool sample was scoured well then impregnated with freshly prepared Ag-NPs colloids under optimized condition and finally rinsed out to remove unfix nanoparticles.

\section{MATERIALS \& METHODS}

\section{Materials}

Wool fabrics were obtained from Jiangsu Shenzhou Woolen Co., Ltd, China. Materials used to synthesize silver nanoparticles were $\mathrm{AgNO}_{3}$ (Shanghai Zhanyun Chemical Co., Ltd), Sodium Alginate (Qingdao Yingfei Chemical Co., Ltd), Sodium Hydroxide (Sinopharm Chemical Co., Ltd). Nonionic commercial detergents were used to wash the silk sample. These materials were used without further purification. Deionized water was used throughout the experiment.

\section{Synthesis of Silver nanoparticles}

The green synthesis of silver nanoparticles was accomplished using an indigenous protocol made in our laboratory. In a brief, $1 \mathrm{mM}$ of $\mathrm{AgNO}_{3}$ was prepared by silver nitrate salt. $1 \% \mathrm{w} / \mathrm{v}$ of sodium alginate was prepared with deionized water. $\mathrm{NaOH}$ solution was prepared by dissolving $\mathrm{NaOH}(0.399 \mathrm{~g})$ in deionized water (100 $\mathrm{ml})$. Typically, $1 \mathrm{ml}$ of sodium alginate $(1 \% \mathrm{w} / \mathrm{v})$ 
solution was dropped in $4 \mathrm{ml}$ of silver nitrate $(0.001 \mathrm{M})$ solution with addition of $\mathrm{NaOH}$ solution (approximately $1 \mathrm{ml}$ of $0.01 \mathrm{M}$ ) to maintain $\mathrm{pH} 11$ and to accelerate the reaction. These mixtures were stirred for 1 minute. Then the mixture is keep inside the heat bath $\left(60^{\circ} \mathrm{C}\right)$ for $40 \mathrm{~min}$. The transparent colorless solution was converted to pale yellow and then into brownish-red color indicated the formation of Ag-NPs. At this end the prepared solution was evaluated by using UV-visible spectra to affirm the production of silver nanoparticles.

\section{Wool functionalization}

The wool fabrics were washed in a bath containing $2 \mathrm{~g} \mathrm{~L}^{-1}$ nonionic detergent with $\mathrm{L}: \mathrm{M}=$ $50: 1$ (liquor to materials ratio) at $50^{\circ} \mathrm{C}$ for $15 \mathrm{~min}$. Then, those were rinsed with distilled water and dried at $110^{\circ} \mathrm{C}$ for $5 \mathrm{~min}$. Green based Ag-NPs were applied on wool surface by using exhaustion methods. The $\mathrm{pH}$ value of Ag-NPs solution was adjusted to 4.0 with acetic acid, and then the scoured wool fabric samples were immersed in an aqueous solution containing Ag-NPs (liquor ratio of $90: 1$ ) at $45^{\circ} \mathrm{C}$ for $2 \mathrm{~h}$. (hereafter referred as "optimized condition") with different concentration

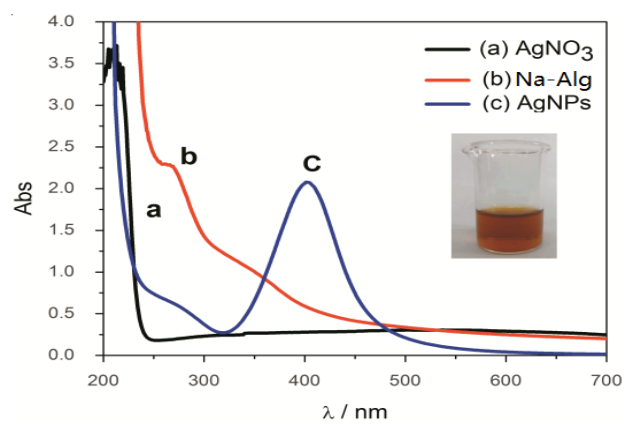

(A)

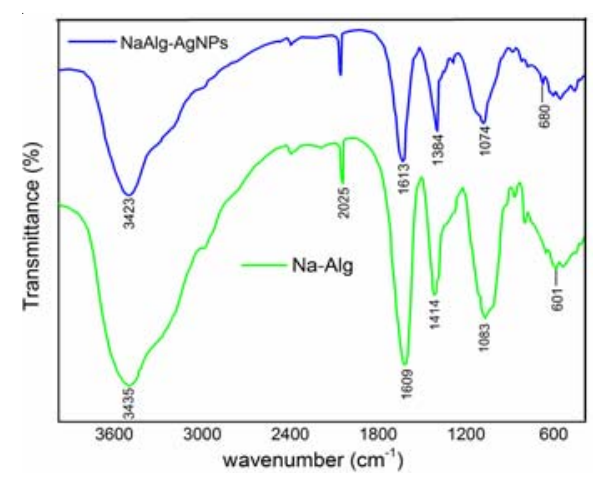

(C) of Ag-NPs. Afterward, the treated wool samples were rinsed with running deionized water.

\section{Measurements and characterization}

The UV-vis spectra of wool fibers with silver nanoparticles were recorded using a Shimadzu 2600 UV spectrophotometer. The morphologies of samples were examined using a scanning electron microscope (SEM) (JEOL, Tokyo, Japan) after gold coating. During SEM test, an EDS spectrum was collected to analyze the chemical elements of the samples. Transmission electron microscopy (TEM) (Hitachi H-7600, Tokyo, Japan) was used to study the particle size distribution of silver nanoparticles. The crystal behavior of samples was obtained using an X-ray diffractometer (XRD) (Bruker, Germany). Fourier transform infrared (FTIR) measurements were performed with a Bruker Tensor 27, Germany in a normal transmission mode. Thermal behavior of samples was performed on a thermogravimetric analyzer (TGA) (Mettler-Toledo Corp., Switzerland) at a heating rate of $10^{\circ} \mathrm{C} / \mathrm{min}$ under nitrogen atmosphere. The color strength $(\mathrm{K} /$ S) and CIELAB color coordinates(under illuminate $D_{65}$ and $10^{\circ}$ standard observer)of samples were

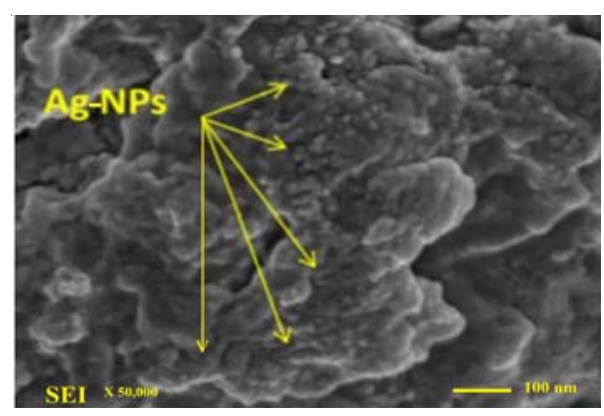

(B)

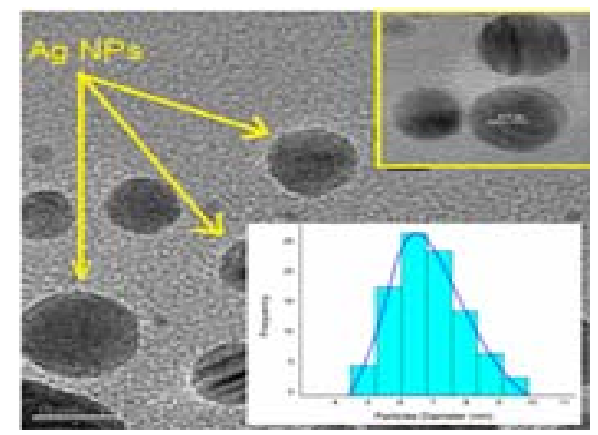

(D) 


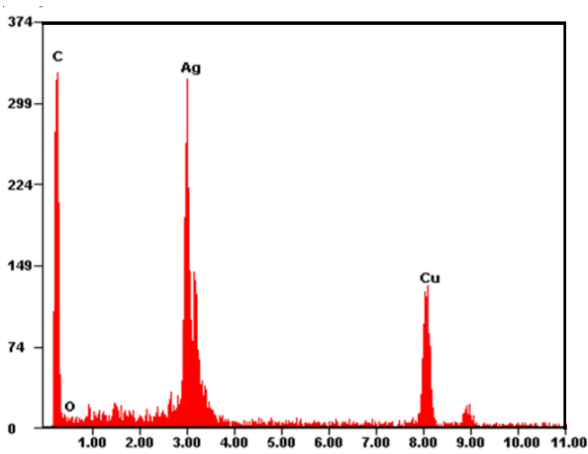

(E)

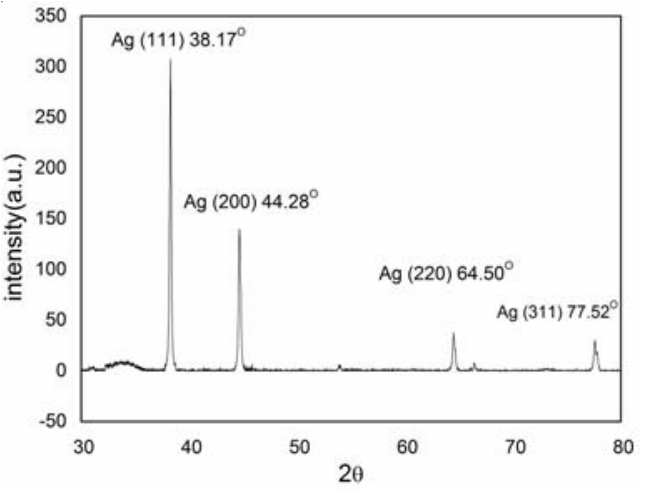

(F)

Fig. 1. (A) UV-vis spectra, (B) FT-IR spectra, (C) SEM, (D) TEM, (E) EDS and (F) XRD of AgNPs

measured with the help of Macbeth Color Eye $7000 \mathrm{~A}$ spectrophotometer. Wool fabrics were tested for the tensile properties before and after Ag-NPs treatment. The crease recovery angle of the samples was determined as per AATCC Test Method 662003 using Sasmira crease recovery tester (India). The stiffness in terms of bending length of untreated and Ag-NPs treated sample was tested as per AATCC Test Method 115-2005 using profile stiffness tester (India).

\section{RESULTS AND DISCUSSION}

\section{Characterization of Ag-NPs}

In the present observe, silver nanoparticles (Ag NPs) had been synthesized in a 'green' method using natural biopolymer alginate with the aid of certainly heating an aqueous combination of sodium alginate and $\mathrm{AgNO}_{3}$. The preliminary affirmation of nanoparticles formation turned into ascertained via recording the absorbance of the colloidal suspension the use of UV-visible spectrophotometry in the range of 200$700 \mathrm{~nm}$ and color change observation. The solution colour turned into reddish brown that indicating reduction of silver ions and appropriate the acknowledged synthesis of AgNPs is apparent in (Fig.1A). The distinctive colors of silver nanoparticles are due to a phenomenon known as plasmon absorbance. Incident light creates oscillations in conduction electrons on the surface of the nanoparticles and electromagnetic radiation is enamored ${ }^{17}$. Fig. 1 A shows the UV-vis spectra of pure silver nitrate $\left(\mathrm{AgNO}_{3}\right)$, sodium alginate $(\mathrm{Na}$ Alg) and AgNPs. In the attendance of Na-Alg, the band at $\sim 301 \mathrm{~nm}$ exhibited by $\mathrm{AgNO}_{3}$ in aqueous solution disappear indicating possible chelation of $\mathrm{Ag}+$ by way of $\mathrm{OH}$ and $\mathrm{COOH}$ groups of alginate. The $\mathrm{Ag}^{+}$chelate produces $\mathrm{Ag}^{\circ}$ on heating. The intensity and role of the shoulder of the alginate spectrum at approximately $290 \mathrm{~nm}$ turned into barely shifted, confirming that there have been interactions among the polymer and the metallic precursor. The UV-Vis spectrum of the Ag-NP showed a characteristic peak at about $400 \mathrm{~nm}$, assigned to a strong surface plasmon resonance has been properly-documented for various metal nanoparticles with sizes ranging from 2 to $100 \mathrm{~nm}^{18}$. For the synthesis of AgNPs, the typically accepted mechanism shows a two-step process, i.e. atom formation and then polymerization of the atoms. In the first step, a portion of metal ions in the solution is reduced by the available reducing groups. The atoms for that reason produced act as nucleation centers and catalyze the discount of the ultimate metal ions in the bulk solution. Compared with other water-soluble polymers, alginate is an anionic polymer with high charge density; the negatively charged alginate allows the appeal of the definitely charged silver ions to the polymeric chains, which have been then decreased by way of the present reducing corporations. The resulting surface negative charge of alginate fragments containing carboxylic groups stabilizes nanoparticles against coalescing with the following one due to electrostatic repulsion and steric outcomes ${ }^{19}$.

The FT-IR measurements were executed out to analyze the functional group of the material. Fig 1B shows the pure Na-Alg spectra presents two primary peaks at about $3435 \mathrm{~cm}^{-1}$ (corresponding to the absorption of stretching of 
the $\mathrm{OH}$ groups)and at $2025 \mathrm{~cm}^{-1}$ (corresponding to the $\mathrm{C} \mathrm{H}$ stretching of the $\mathrm{CH}_{2}$ groups). The $\mathrm{CO}$ asymmetrical stretching at $\max 1609 \mathrm{~cm}^{-1}$ along with a weaker symmetrical stretching band at $1414 \mathrm{~cm}^{-1} 20$ were empiric due to salt nature of carboxylic acid groups at pure Na-Alg. An accelerated band at $1083 \mathrm{~cm}^{-1}$ is apery the stretching of $\mathrm{C}-\mathrm{O}-\mathrm{C}$ group; the peaks at $601 \mathrm{~cm}^{-1}$ belong to $\mathrm{C}-\mathrm{O}-\mathrm{C}$ glycosidic linkage (ring breathing) ${ }^{21}$. They are accompanying to its saccharide structure ${ }^{22}$. In the case of AgNPs, the band of $\mathrm{CO}_{2}$ " is shifted to $1613 \mathrm{~cm}^{-1}$ in addition the peaks of 3435 and $1414 \mathrm{~cm}^{-1}$ shift to 3422 and
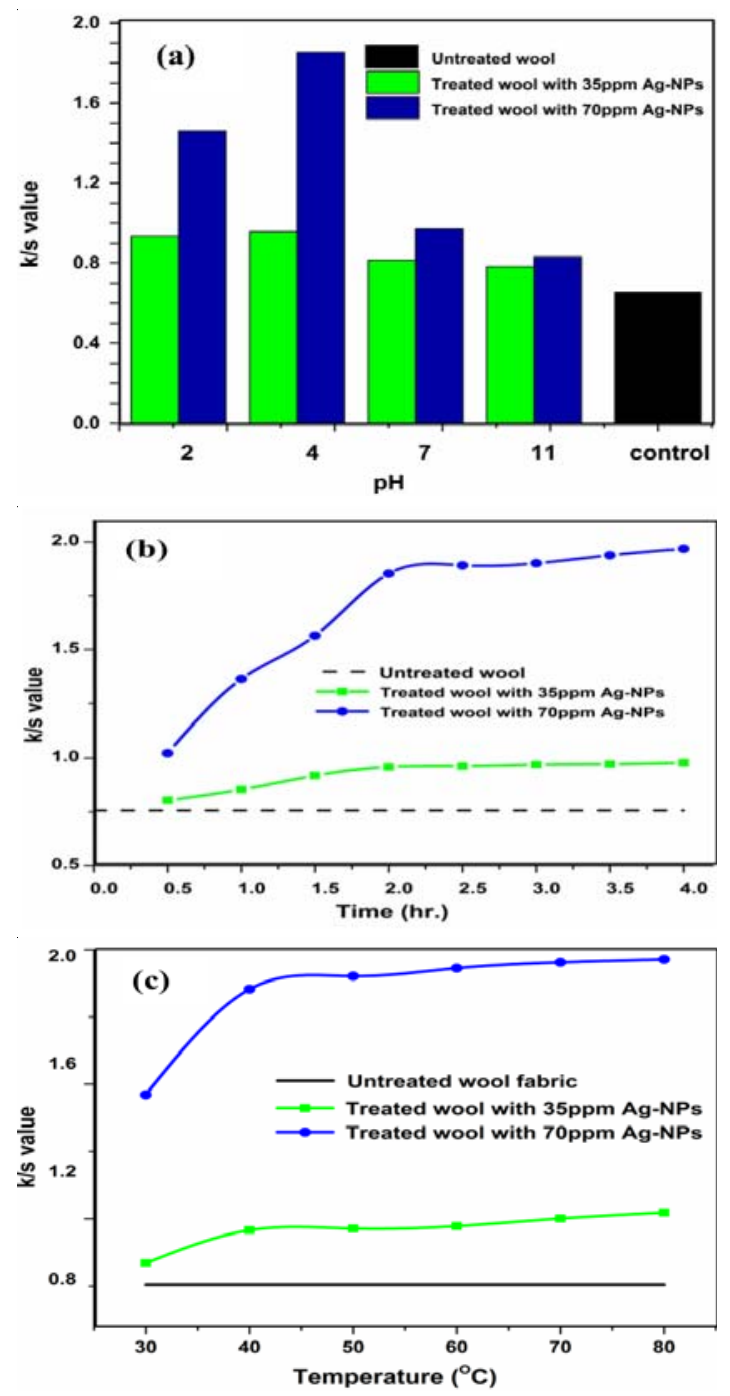

Fig. 2. Effect of the (A) pH (time $40 \mathrm{~min}$, temp $40^{\circ} \mathrm{C}$ ), (B) temperature ( $\mathrm{pH} \mathrm{4,} \mathrm{time} 40 \mathrm{~min})$ and $(\mathrm{C})$ Effect of the application time $\left(\mathrm{pH} 4\right.$, temp $\left.40^{\circ} \mathrm{C}\right)$ on the particle uptake in terms of $\mathrm{K} / \mathrm{S}$ value
$1384 \mathrm{~cm}^{-1}$ due to ring stretching of metal groups and indicating that $\mathrm{Na}-\mathrm{Alg}$ was doped by $\mathrm{NO}_{3}^{-}$ because of the stabilization of Ag-NPs. The comparison of FTIR spectra between the Na-Alg and Na-Alg/Ag-NPs showed only minor changes in the position as well as the absorption bands. Thereby, FT-IR spectrum confirms that Ag-NPs have been capped with the aid of the lone pair electrons around the oxygen atoms in organic compound in $\mathrm{Na}-\mathrm{Alg}$ with van der waals interaction forces ${ }^{23}$. Fig $1 \mathrm{C}$ presents SEM image of Na-Alg loaded Ag-NPs. It is manifest that silver nanoparticles synthesized by greenly can cautiously be admired as nanoparticles with spherical nature. The agglomeration of silver nanoparticles was due to interactions of hydrogen bond and electrostatic interactions between the bioorganic capping molecules apprenticed to the AgNPs ${ }^{24}$. TEM image with the corresponding particle size distribution (PSD) of the prepared Ag-NPs is shown in Fig 1D. It demonstrates the formation of Ag-NPs and offers us clean view of shape, length and distribution of the particles in nanoscale. From the image, it could be seen that the Ag NPs located are round in form and nicely separated in aqueous medium which are covered via layer. The layer can be the phytoconstituents of sodium alginate. In addition, the aggregation is lower because of less collision of silver nanoparticles. From the scale distribution picture, it could be ascribed that the maximum number of nanoparticles are inside the length among 6 to $10 \mathrm{~nm}$. The reductive properties of sodium alginate are notably more desirable owing to the base hydrolysis with the formation of low molecular weight reducing fragments, and consequently, reflecting the twin function of sodium alginate as stabilizing and reducing agent in alkaline medium ${ }^{25}$. The additional support of reduction of $\mathrm{Ag}^{+}$ions to elemental silver was confirmed by EDS analysis is shown in Fig 1E. The optical absorption peak is observed approximately at $3 \mathrm{keV}$, which is typical for the absorption of metallic silver nanocrystalline due to surface plasmon resonance ${ }^{26}$, which confirms the presence of nanocrystalline elemental silver. An XRD pattern of Na-AgNPs become completed to verify the crystal phase of the prepared AgNPs within the range of $30-80^{\circ}$. Fig. $1 \mathrm{~F}$ suggests typical XRD pattern of the alginate-AgNPs prepared with several distinct diffraction peaks at approximately $38.1^{\circ}, 44.2^{\circ}, 64.3^{\circ}$ 
and $77.4^{\circ}$ are assigned to reflections from the (111), (200), (220), and (311) planes of the silver crystal, respectively, which confirms the existence of silver and further on the basis that they can be indexed as face-centered-cubic (FCC) structure of silver. These peaks are due to the crystalline and amorphous natural stages, accompanying crystallized AgNPs. In addition to the Bragg peaks adumbrative of silver nanocrystals, added peaks were aswell observed, although they were not assigned to the spectrum and may accept been due to amoebic compounds, responsible for silver ion reduction and the stabilization of the resultant nanoparticles ${ }^{27}$.

\section{Optimization of treatment conditions}

Fig.2(a) represents the effect of $\mathrm{pH}$ on color strength (K/S) values of wool treated by 35 and 70 ppm Ag-NPs conc. with respect to controlled conditions. When the $\mathrm{pH}$ of Ag-NPs solution was 2, the solution was approximately colorless after treatment of wool fabrics and the assembling trend of Ag-NPs was the same as that at $\mathrm{pH} 4$. But still better result observed when $\mathrm{pH}$ of the application medium 4 than $\mathrm{pH} 2$ in terms of K/S value. From the Fig.2(a), it can be depicted that maximum color strength $(\mathrm{K} / \mathrm{S})$ value obtained at $\mathrm{pH} 4$, a significant proportion of internal amino groups are protonated, leading to a neutralization of this surface charge; however, the carboxylate anions are not substantially protonated until the $\mathrm{pH}$ approaches high acidic medium. This is the reason for the use of acid when dyeing wool with highly hydrophilic leveling acid dyes and it is clear from Fig.2(a) that Ag-NPs behave in a similar manner to those dyes ${ }^{28}$. However, the Ag-NPs deposition did not occur when the application medium was approximately neutral condition like $\mathrm{pH} 7$ as there is no significant change in $\mathrm{K} / \mathrm{S}$ value. Similar phenomenon has been observed when the solution was in highly alkaline medium like $\mathrm{pH} 11$. In addition, the $\mathrm{pH}$ value played a vital contribution in the Ag-NPs treatment process because the different surface characteristics of wool polymer under different $\mathrm{pH}$ values. So it could be concluded that the Ag-NPs on wool surface is applicable may be due to the electrostatic interaction between carboxylate groups of Ag-NPs and amino groups of wool polymer.

Optimization of treatment time can be an important step in sustainable textile functionalization with respect to the energy savings. Fig.2(b) shows the progress of wool treatments with different concentration of Ag-NPs at times ranging from $30 \mathrm{~min}$. to $4 \mathrm{~h}$. in terms of K/S value. The result revealed that, the $\mathrm{K} / \mathrm{S}$ value increases from $30 \mathrm{~min}$. to $2 \mathrm{~h}$. time interval. This result can be attributed due to the changing pattern of the wool materials and the zeta potential of the Ag-NPs. Because the wool surface having positive charge and Ag-NPs surface having negative charge resulting a strong attraction between them ${ }^{29}$. Therefore, up to $2 \mathrm{~h}$. of exhaustion time, the rate of Ag-NPs uptake by wool was very high; however, there was no significant change in curve but asymptotic behaviour with more exhaustion time beyond 2 hours. The application of Ag-NPs on wool fabrics can be controlled by the temperature of application medium. The Fig 2 (c) evident that the rate of Ag-NPs uptake by wool samples were increased up to $40^{\circ} \mathrm{C}$ temperature and thus completed in 2 hours. An interesting correlation between temperature and time was observed in this experiment. When the temperature was increased to $85^{\circ} \mathrm{C}$, the deposition was finished in about $1 \mathrm{hr}$ at same concentration because of higher kinetic energy of the system at higher temperature ${ }^{30}$.

\section{Absorption characteristics}

The UV-Vis absorption spectrum of the solutions of silver nanoparticle before and after coating was presented in Fig.3(A). It can be acknowledge that, 70 ppm AgNPs solution absorption intensity is higher than 35 ppm AgNPs solution after coating. This could be due to the high

Table. 1: Color coordinates data of untreated and Ag-NPs treated wool fabrics

\begin{tabular}{lccccc}
\hline Sample & CIE DE & CIE L* & CIE a* & CIE b* & K/S value \\
\hline Untreated wool & 0.76 & 82.97 & 0.70 & 14.55 & 0.7556 \\
Treated wool with 35ppm Ag-NPs & 7.47 & 76.45 & 3.36 & 15.05 & 0.9577 \\
Treated wool with 70ppm Ag-NPs & 17.81 & 67.52 & 4.80 & 21.05 & 1.8529 \\
\hline
\end{tabular}


affinity of wool material that's enough sufficient to adsorb nearly all $\mathrm{Ag}^{+}$and $\mathrm{Ag}$ NPs. The emerged colours of the treated fabrics had been associated with the surface plasmon resonance (SPR) properties of green synthesized AgNPs on wool fabrics. In order to analyze the SPR of AgNPs on dealt with fabric, the absorption traits of the 35 and $70 \mathrm{ppm}$ AgNPs treated fabrics had been evaluated using a spectrophotometer within the variety of 200$800 \mathrm{~nm}$ is shown in Fig. 3(B) and illustrate that, with the increasing of Ag-NPs concentration on the wool surface, the absorbency increased and the main plasmon peak was observed around $425 \mathrm{~nm}$. Also, the intensity of the observed plasmon peaks was increased with raising the Ag-NPs concentration. Another prominent peak of untreated wool at 460 $\mathrm{nm}$ gradually disappears with the increasing of $\mathrm{Ag}$ NPs concentration. It is assumed that, this because of the interaction of the very small Ag-NPs with the wool polymer system.

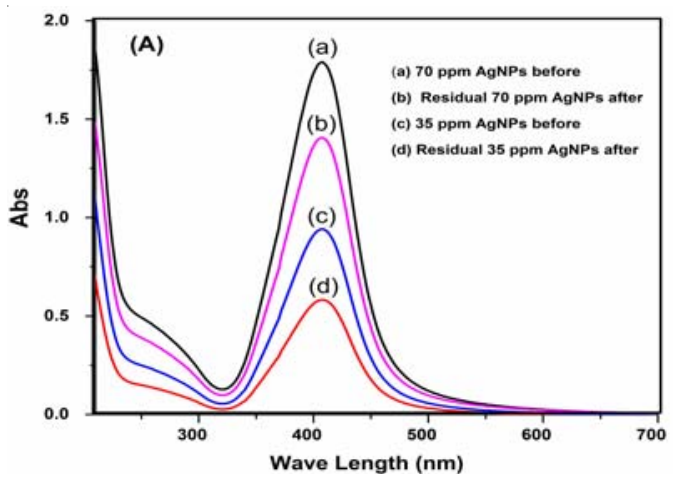

(a)

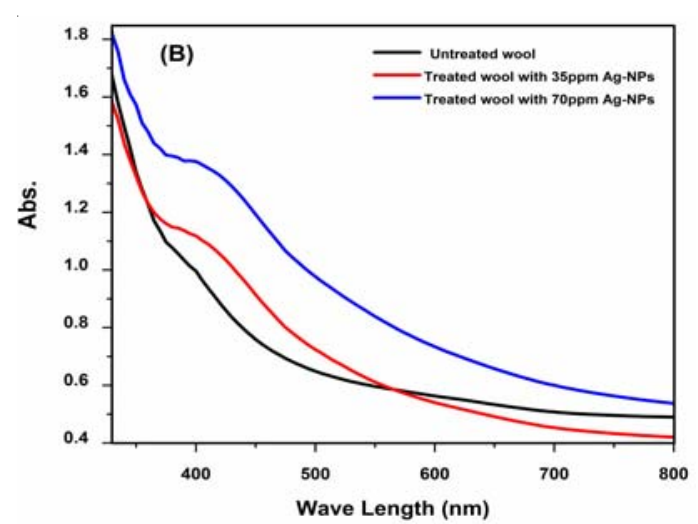

(b)

Fig. 3. UV-vis spectra (A) For residual Ag NPs solution with before and after treatment and (B) The untreated and Ag NPs treated wool fabrics.

\section{Color measurements}

The treated wool with Ag-NPs exhibits higher $\mathrm{K} / \mathrm{S}$ value than the untreated wool sample due to the adsorption of Ag-NPs (Table 1). It was described that $\mathrm{K} / \mathrm{S}$ value can be a measure of deposited Ag-NPs concentration on fabric. The K/S is the ratio between the absorption $(\mathrm{K})$ and scattering coefficient (S) of the treated materials. However, the K/S value of the treated wool fabric has different trend comparing to the Ag content. Ag-NPs absorption considerably depends on the shape, size and application media ${ }^{31}$ and varying synthesizing condition can influence these characteristics $^{32,}{ }^{33}$. Therefore, increasing the concentration of Ag-NPs can effect on the absorption features of the loaded Ag-NPs but may not ensure any correlation between the color strength of the treated silk and $\mathrm{Ag}$ content.

\section{Color s trength of the treated silk and Ag content.}

As shown in Fig.4 the wool fabrics appeared in vivid colors derived from the LSPR of silver NPs. However, the colors of these treated wool fabrics were slightly different from that of original silver NP solutions, which is due to the environment changes around silver NPs from water to the interface of air and wool fiber ${ }^{34}$.

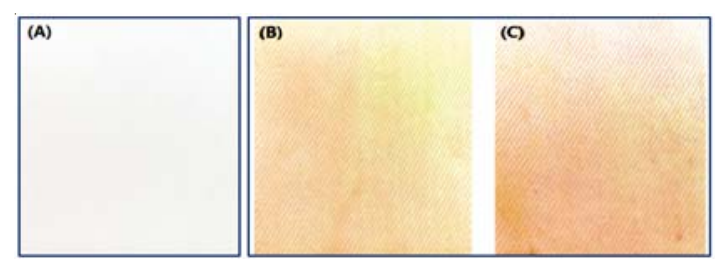

Fig. 4. Photographs of untreated (A) 35ppm (B) and 70ppm (C) Ag-NPs under optimized condition

\section{FT-IR Spectra}

Figurs. 5 shows the FT-IR transmission spectra of the untreated wool and AgNPs treated wool fabrics. Wool is composed of a cuticle and cortex, whereas the cortex makes up the main portion of the wool. It is made up of more than 18 amino acids, which can be alienated into four distinct groups: cationic, anionic, nonpolar, and polar. The main functional groups include carboxyl $(-\mathrm{COOH})$, amino $\left(-\mathrm{NH}_{2}\right)$, and hydroxyl $(-\mathrm{OH})$ groups $^{35}$. Overall, as shown in Fig.5, all wool fibers exhibited similar absorption at the following wavelengths: $3260 \mathrm{~cm}^{-1}(\mathrm{~N}-\mathrm{H}$ and $\mathrm{O}-\mathrm{H}), 2881 \mathrm{~cm}^{-1}$ $\left(-\mathrm{CH}_{2}\right), 1670 \mathrm{~cm}^{-1}$ (amide I), $1540 \mathrm{~cm}^{-1}$ (amide II), and $1270 \mathrm{~cm}^{-1}$ (amide III). When spectra of untreated 
Fig. 5(a) and treated Fig. 5(b and c) were compared, new peaks at $1260 \mathrm{~cm}^{-1}$ and $1100 \mathrm{~cm}^{-1}$ were observed. The intensity of these peaks is stronger than the peaks for the spectrum of untreated wool, due to interaction between the $\mathrm{OH}$ of sodium alginate and the amide bond of the wool. This suggests that the Ag-NPs were successfully adsorbed on wool and confirms that the two new peaks in spectra are derivative from the interaction of the wool polymer system and Ag-NPs ${ }^{36}$.
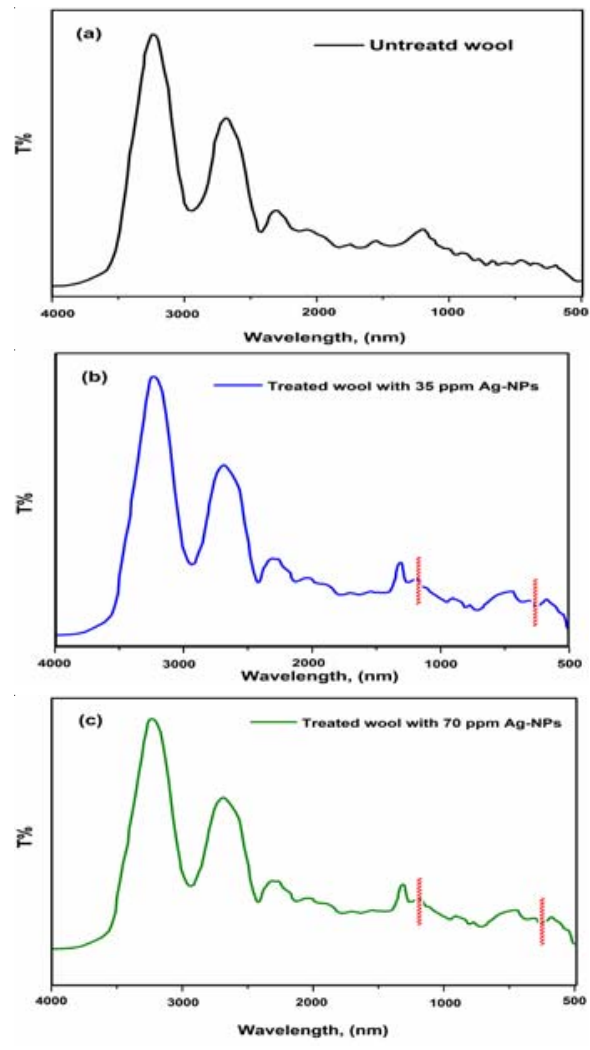

Fig. 5. FT-IR spectra of samples

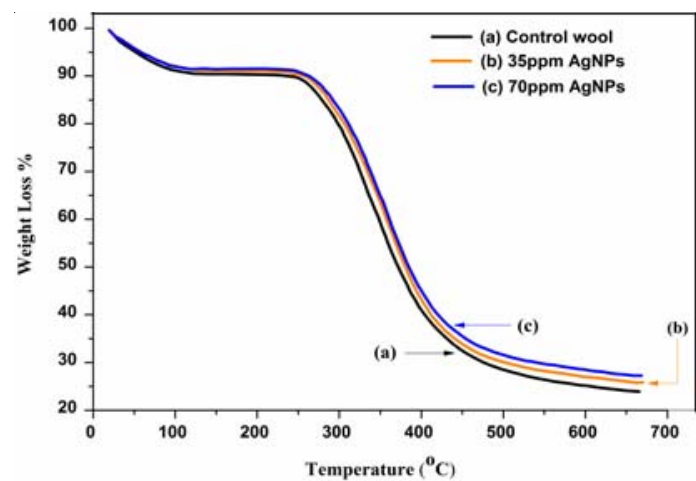

Fig. 6. TGA curve of samples

\section{Thermogravimetric Analysis (TGA)}

Thermogram of weight loss in percentage of wool fabric by heating was recorded and compared with treaded wool with different concentration of Ag-NPs are illustrated in Fig 6. The untreated (blank) wool fabric after the loss of regains water (occurring from $30^{\circ} \mathrm{C}$ to $160^{\circ} \mathrm{C}$ and is accompanied by a decrease of $7 \%$ in wool fibers mass and is ascribed to the loss of water), the initial thermocracking reaction begins at approximately $280^{\circ} \mathrm{C}$ and the weight became reduced. This is because of destruction of disulphide linkages and the elimination of $\mathrm{H}_{2} \mathrm{~S}$, followed by the thermal pyrolysis of the chain linkages, peptide bridges and some other lateral chains, which finally leads to backbone degradation ${ }^{37,38}$. On the other hand, wool fabrics treated with Ag-NPs were found to have a delayed thermocracking reaction. So, the Ag-NPs treating of wool fabrics lead to increase its thermal stability, which is assured the consequence of AgNPs in advanced treating effectiveness. This is because sodium alginate \& silver nano composite may be act as cross linker or filler of wool polymer system; therefore, heat has significant effect on the treated fabrics. So, we can conclude the untreated wool having a higher weight loss, while the highest Ag-NPs concentration offerings the lowest weight loss. The results are in consensus with the results of silver content.

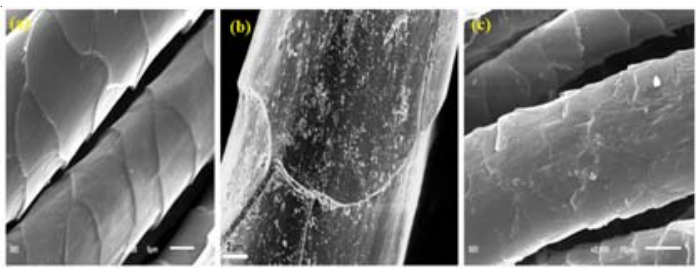

Fig. 7. SEM images of (a) control wool fibers (b) $35 \mathrm{ppm}$ and (c) 70ppm Ag-NPs

\section{SEM Analysis}

SEM analysis were done to observe the surfaces morphologies of untreated and treated wool fabrics with different concentration of Ag-NPs. Fig. 7 display the SEM characterization of wool fabrics before and after treatment with Ag-NPs. A clear and distinctive scaled assembly of a wool fiber can be detected in the case of control sample. It comprises of cuticle cells covering to form a pattern like that of tiles on a roof ${ }^{39}$. The result shows a high level of heterogeneity of the surface covering; namely, certain areas are tightly covered, whereas 
in others, a thinly covered pattern can be detected. The presence of Ag-NPs on the surface of treated wool fibers indicate that the Ag-NPs were successfully adsorbed on the wool fibers. There were silver clusters on the surface of the wool fibers. Some of them were well separated when the concentration of Ag-NPs was 35ppm due to the protection by $\mathrm{Na}-\mathrm{Alg}$, but larger aggregates can also be observed in the case of 70ppm. These aggregations create a smooth outer layer of wool fibers which suggest a possible layer by layer nanocoating technique for multifunctional application.

\section{Functional properties}

The significance of Ag-NPs on tensile features, crease recovery performance and bending properties has been examined. It was noticed from the Table 2 that the treatment of $\mathrm{Ag}$ NPs in to the wool polymer results in progression in the load bearing capacity of the fiber. According to this consequence, it can be determined that the Ag-NPs treated wool sample amplifies its mechanical behavior. Because of the particle size in nano range, Ag-NPs having enough capability to enter in between the wool polymer system and possibly act as a filler or crosslinking agent which also guarantee to the load shearing ability during the load application to the material. There is little improvement in crease recovery angle of Ag-NPs treated wool fabric with a little increase in bending length. This phenomenon ensures that the particle entered in between the polymer molecules do not interface much to the polymer flexibility of the system. The behavior is thus devoid of harshness to the materials. No distinct change can be felt in hand feeling between untreated and Ag-NPs treated wool fabrics; all the wool fabrics feel soft and smooth equally. To further observe the influences of the assembly of Ag-NPs on handle of the wool fabrics, bending lengths were measured (Table 2).

Table. 2: Physical and mechanical properties of samples

\begin{tabular}{lccccc}
\hline Properties Untreated wool & 35ppm Ag-NPs & \% change & 70ppm Ag-NPs & \% change \\
\hline BKL & 6.32 & 6.42 & +1.58 & 6.49 & +2.69 \\
CRA & 140 & 147 & +5.00 & 150 & +7.14 \\
BL & 2.08 & 2.18 & +4.81 & 2.22 & +5.71 \\
\hline BKL
\end{tabular}

Since fabric bending length is related to fabric stiffness or handle, these results propose that the assembly of Ag-NPs did not change the handle of wool fabrics, due to the size of Ag-NPs with in nano range. Wool fabrics are hydrophobic in nature. The surface assembling and modification can change the wettability of fabrics, which can ensure better comfortableness of textile. In this study, the adsorption ability of the wool fabrics to Ag-NPs colloid was measured by increasing weight ratios of Ag-NPs colloid to wool. All the Ag-NPs in the colloid were nearly assembled on the wool when the ratio was changed to 500 from 100 , which can be inferred from extinction spectrum of Ag-NPs colloid after treatment (data not shown). Furthermore, to evaluate the stability of the Ag-NPs on the wool with respect to K/S value of the wool fabric before and after placement for a period of time were recorded and compared. These results exhibit that the wool fabrics treated with Ag-NPs possess good stability, which is substantial for industrial application of treatment with Ag-NPs. It is supposed that the oxygen in air did not oxide the Ag-NPs assembled on wool fabric though the morphologies of the Ag-NPs in colloid can be converted by assistance of dissolved oxygen in solution under heating ${ }^{40}$, which is possibly attributed to the change of neighboring medium around Ag-NPs.

\section{CONCLUSION}

In this research, we have demonstrated the Ag-NPs could be successfully bonded to the wool fibre surface with appropriate surface modificationby physical \& chemical interactions. In particular, it is maximal at low $\mathrm{pH}$ in which there are very few anionic groups on the wool fiber; the NPs also need to have sufficient charge to maintain their stability as dispersion. FT-IR characterizations demonstrated that the Ag-NPs were effectively assembled on the wool fibres. SEM analysis 
confirmed that the industrial process to obtain fabrics did not alter the strong adhesion of Ag-NPs to the fibers and their uniform distribution. The produced silver-treated wool fabrics maintained the high flexibility and elasticity typical of wool. The stability of the Ag-NPs treatment is satisfactory: even after several cycles of thermal treatments. AgNPs endowed the wool fabrics with different colors which suggest eliminate the limitation of traditional wool dyeing process. We also suggest that this product can serve to functionalize fabric with the high performance. It has been shown that nanotechnology and textiles can be combined to develop novel fabrics with technical multi-functions, which can facilitate extensive applications of nanoparticles in textiles.

\section{ACKNOWLEDGEMENT}

The authors wish to thanks the Runhe 1chemical industry, china \& Color root (Hubei) technology limited, china, for providing the technical support and the school of chemistry \& chemical engineering, Wuhan Textile University, china, for providing chemicals and all measurements.

\section{REFERENCES}

1. Whitesides, G. M., Small, 2005, 1, 172-179.

2. Yetisen, A. K., Qu, H., Manbachi, A., Butt, H., Dokmeci, M. R., Hinestroza, J. P., Skorobogatiy, M., Khademhosseini, A., and Yun, S. H., ACS nano, 2016, 10, 3042-3068.

3. Qian, L., AATCC rev, 2004, 4, 14-16.

4. Bozzi, A., Yuranova, T., and Kiwi, J., J. of Photochem. and Photobio. A: Chem., 2005, 1,72 27-34.

5. Avila, A. G. and Hinestroza, J. P., Nat. nanotech., 2008, 3 , 458-459.

6. Zhao, G. and Stevens, S. E., Biometals, 1998, 11, 27-32.

7. Iravani, S., Korbekandi, H., Mirmohammadi, S., and Zolfaghari, B., Res. in phar. sci., 2014, 9, 385.

8. Bonilla, J. J. A., Guerrero, D. J. P., Sáez, R. G. T., Ishida, K., Fonseca, B. B., Rozental, S., and López, C. C. O., J. of Nanosci. and Nanotech., 2017,17, 1729-1739.

9. Sarsar, V., Selwal, K. K., and Selwal, M. K., J. of Microbio. and Biotech. Res., 2017, 3 , 27 32.

10. Poliakoff, M., Fitzpatrick, J. M., Farren, T. R., and Anastas, P. T., Science, 2002, 297, 807810.

11. Raveendran, P., Fu, J., and Wallen, S. L., J. of the Ame.. Chem. Soci., 2003, 125, 1394013941.

12. Maclaren, J. A. and Milligan, B. , Marrickville, Australia: Science Press., 1981, 122.

13. Pollini, M., Paladini, F., Licciulli, A., Maffezzoli, A., Nicolais, L., and Sannino, A., J. of App. Poly. Sci., 2012, 125, 2239-2244.
14. Sepahi Rad, P., Montazer, M., and Karim Rahimi, M., J. of App. Poly. Sci., 2011, 122, 1405-1411.

15. Ki, H.Y., Kim, J. H., Kwon, S. C., and Jeong, S. H., J. of Mat. Sci., 2007, 42, 8020-8024.

16. Montazer, M., Behzadnia, A., Pakdel, E., Rahimi, M. K., and Moghadam, M. B., J. of Photochem. and Photobio. B: Biology, 2011, 103, 207-214.

17. Slistan-Grijalva, A., Herrera-Urbina, R., Rivas-Silva, J., Ávalos-Borja, M., CastillónBarraza, F., and Posada-Amarillas, A., Phy. E: Low-dim. Sys.and Nanostru., 2005, 27, 104-112.

18. Stamplecoskie, K. G. and Scaiano, J. C., J. of the Ame. Chem. Soci., 2010, 132, 1825-1827.

19. Abdel-Halim, E. and Al-Deyab, S. S., Carbo. Poly., 2011, 86, 1615-1622.

20. Manuja, A., Kumar, S., Dilbaghi, N., Bhanjana, G., Chopra, M., Kaur, H., Kumar, R., Manuja, B. K., Singh, S. K., and Yadav, S. C., Nanomedicine, 2014, 9, 1625-1634.

21. CardenasJiron, G., Leal, D., Matsuhiro, B., and OsorioRoman, I., J. of R. Spectro., 2011, 42, 870-878.

22. Sartori, C., Finch, D. S., Ralph, B., and Gilding, K., Polymer, 1997, 38, 43-51.

23. Mandal, A., Sekar, S., Chandrasekaran, N., Mukherjee, A., and Sastry, T. P., RSC Adv., 2015, 5, 15763-15771.

24. Devaraj, P., Kumari, P., Aarti, C., and Renganathan, A., J. of nanotech., 2013, 2013.

25. El-Rafie, M., El-Naggar, M., Ramadan, M., Fouda, M. M., Al-Deyab, S. S., and Hebeish, 
A., Carbo. Poly., 2011, 86, 630-635.

26. Mohammed Fayaz, A., Balaji, K., Girilal, M., Kalaichelvan, P., and Venkatesan, R., J. of Agr. and F. Chem., 2009, 57, 6246-6252.

27. Roopan, S. M., Madhumitha, G., Rahuman, A. A., Kamaraj, C., Bharathi, A., and Surendra, T., Ind.Crop. and Prod., 2013, 43, 631-635.

28. Briggs, T. and Bull, A., The J. of Phy. Chem., 1922, 26, 845-875.

29. Tang, B., Wang, J., Xu, S., Afrin, T., Xu, W., Sun, L., and Wang, X., J. of coll. and int. sci., 2011, 356, 513-518.

30. King, D. G. and Pierlot, A. P., Color. Tech., 2009, 125, 111-116

31. Cobley, C. M., Skrabalak, S. E., Campbell, D. J., and Xia, Y., Plasmonics, 2009, 4, 171-179.

32. Mulvaney, P., Langmuir, 1996, 12, 788-800.
33. Shin, H. S., Yang, H. J., Kim, S. B., and Lee, M. S., J. of coll. and int. sci., 2004, 274, 89-94.

34. Hu, M., Chen, J., Marquez, M., Xia, Y., and Hartland, G. V., The J. of Phy. Chem. C, 2007, 111, 12558-12565.

35. Hsieh, S. H., Huang, Z., Huang, Z., and Tseng, Z., J. of App. Poly. Sci., 2004, 94, 1999-2007.

36. Bose, P. P., Drew, M. G., and Banerjee, A., Org. lett., 2007, 9 , 2489-2492.

37. Davies, P. J., Horrocks, A. R., and Miraftab, M., Poly. int., 2000, 49, 1125-1132.

38. Forouharshad, M., Montazer, M., Moghadam, M. B., and Saligheh, O., Ther. Acta, 2011, 516, 29-34.

39. Gallico, L. L., Vigliano Biellese, Italy, 2000.

40. Tang, B., Xu, S., Hou, X., Li, J., Sun, L., Xu, W., and Wang, X., ACS app. mat. \& int., 2013, 5, 646-653. 